Artículo

\title{
Efecto de la fertilización orgánica e inorgánica en la producción y calidad de Brachiaria brizantha
}

\author{
Wilgince Apollon ${ }^{1,2 \S}$ \\ Yviane Jean-Baptiste ${ }^{1}$ \\ Birmania J. Wagner ${ }^{1}$ \\ Alejandro Isabel Luna-Maldonado ${ }^{2}$ \\ Héctor Silos-Espino ${ }^{3}$
}

${ }^{1}$ Facultad de Ciencias Agronómicas y Veterinaria-Universidad Autónoma de Santo Domingo. Camino Engombe s/n, Santo Domingo Oeste, República Dominicana. CP. 10904. Tel. +1809 5318600. (yvianejb@gmail.com; birmaniawagner@yahoo.com). ${ }^{2}$ Facultad de Agronomía-Departamento de Ingeniería Agrícola y de los Alimentos-Universidad Autónoma de Nuevo León. Francisco Villa s/n, ExHacienda El Canadá, General Escobedo, Nuevo León, México. CP. 66050. Tel. 8113404399. (alejandro.lunaml@uanl.edu.mx). ${ }^{3}$ TecNM-Campus Instituto Tecnológico El Llano Aguascalientes. Carretera Aguascalientes-SLP km 18, El Llano, Aguascalientes, México. CP. 20330. Tel. 4499161251. (silosespino@hotmail.com).

${ }^{\S}$ Autor para correspondencia: wilgince.apollon@uanl.edu.mx.

\section{Resumen}

El rendimiento y la composición bromatológica de dos cultivares de Brachiaria brizantha (Sinaí y Xaraés), en dos tipos de suelo y tres tipos de fertilizante, se evaluaron mediante un diseño completamente al azar con arreglo factorial $(2 \times 2 \times 3)$ con cuatro repeticiones. Las variables estudiadas fueron: rendimientos de materia verde, materia seca, número de hojas, así como la altura de planta, los contenidos de proteína cruda, calcio, fósforo y fibra cruda. El factor fertilizante tuvo efecto positivo sobre la variable altura de la planta. Con la aplicación del estiércol de bovino se obtuvo la altura promedio más alta de $96.06 \mathrm{~cm}$. A los 21 días, se obtuvo el rendimiento más alto de materia verde de $756.2 \mathrm{~kg} \mathrm{ha}^{-1}$ con el fertilizante mineral. El contenido de materia seca fue influenciado significativamente por la interacción triple $(p \leq 0.05)$, donde Sinaí logró el valor promedio más alto de $132.5 \mathrm{~kg} \mathrm{ha}^{-1}$ a los 21 días de rebrote. El mayor contenido promedio de PC se encontró en las plantas de ambas variedades que recibieron fertilización química. El mayor contenido promedio de Ca se encontró en Sinaí (1.9\%) y Xaraés (2.08\%) que recibieron fertilización orgánica. Mientras que el mayor contenido promedio de $\mathrm{P}$ fue encontrado en Xaraés $(0.42 \%)$ con la aplicación de HMA. Finalmente, la planta Sinaí que recibió estiércol de bovino en suelo esterilizado presentó mayor contenido promedio de FC (27.27\%). La fertilización orgánica e inorgánica tuvo un efecto significativo en el rendimiento y la calidad nutricional de los pastos.

Palabras claves: Brachiaria brizantha, composición bromatológica, rendimiento.

Recibido: noviembre de 2021

Aceptado: febrero de 2022 


\section{Introducción}

Los pastos son una fuente de componentes esenciales, accesibles y económicos en la producción ganadera, que generalmente se utiliza en el sector ganadero para la alimentación de los animales. Sin embargo, para obtener el mejor beneficio de éstos, es necesario que sean de buena calidad nutritiva. Según Lok et al. (2017), las especies forrajeras han tenido un gran impacto en climas cálidos, por ser un alimento de costo muy bajo, así como también por su aporte en cuanto a los requerimientos proteínicos en la dieta animal. Las especies Brachiaria son pastos forrajeros importantes en países tropicales y subtropicales (Bogdan, 1977). Éstas son gramíneas perennes provenientes de África tropical, de reciente introducción a la Amazonía ecuatoriana.

Estas especies de forraje tienen crecimientos erectos y semi-erectos, con raíces profundas de color blanco amarillento y de consistencia blanda. La inflorescencia es una panícula de $40 \mathrm{a} 50 \mathrm{~cm}$ de longitud, generalmente con cuatro racimos de 8 a $12 \mathrm{~cm}$ y una sola hilera de espiguillas sobre ellos; crecen en suelos de mediana a alta fertilidad y requieren precipitaciones de 1000 a $3500 \mathrm{~mm}$ anuales. De acuerdo con Reyes et al. (2009), la producción de forraje de los cultivares de Brachiaria varía mucho entre cultivares. Se ha reportado un rendimiento promedio de materia seca (MS) total de $6.34 \mathrm{t} \mathrm{ha}^{-1}$ a las 10 semanas de rebrote en Brachiaria spp. (Garay et al., 2017), también se encontró que la digestibilidad de MS en B. brizantha $c v$ Toledo varió de $67 \%, 64 \%$ y $60 \%$ a los 25, 35 y 45 días, respectivamente (Lascano et al., 2002).

A esas edades, la producción anual varió entre 8 y 20 t de $\mathrm{MS}^{-1}$. Por otro lado, el cultivar humidicola presentó una digestibilidad de MS de 55\% (Da Silva-Souza et al., 1992; Moura et al., 2002). El $c v$ Toledo alcanzó concentraciones de proteína cruda (PC) en las hojas de 13\%, 10\% y $8 \%$ a edades de rebrote a los 25, 35 y 45 días respectivamente. El valor nutritivo de los pastos depende de la edad de las especies y la fertilización, especialmente el aporte de nitrógeno (N). El $\mathrm{N}$ aplicado en condiciones favorables para el crecimiento vegetal, proporciona una mayor producción de MS y la producción de proteínas a partir de carbohidratos (Havlin et al., 2005).

La fertilización de los pastos Brachiaria, es una de las prácticas de mayor impacto sobre los incrementos en la producción de biomasa y sobre los niveles de proteína, lo cual también ha mejorado la calidad del forraje. Por lo anterior, se han utilizado varios tipos de fertilizantes tales como: estiércol de bovino, fertilizante mineral (Jiménez et al., 2010) y también hongos micorrízicos arbusculares (HMA) (González et al., 2011), para aumentar el rendimiento de los pastizales. El uso de estos fertilizantes (orgánico e inorgánico) aumenta la fertilidad de los suelos ácidos (Jiménez et al., 2010). Para mejorar la calidad de los pastos, es necesario aplicar una fertilización adecuada. El objetivo de este estudio fue evaluar los componentes el rendimiento y la composición bromatológica de dos cultivares de B. brizantha (Sinaí y Xaraés), mediante la aplicación de tres tipos de fertilizantes (hongo micorrízico arbuscular, estiércol de bovino y fertilizante mineral).

\section{Materiales y métodos}

\section{Ubicación y descripción del área experimental}

El estudio se llevó a cabo durante cinco meses (marzo a julio de 2016) en el vivero experimental de la Facultad de Ciencias Agronómicas y Veterinarias (AGROVET) de la Universidad Autónoma de Santo Domingo (UASD), ubicada en la latitud $18^{\circ} 27^{\prime} 27^{\prime \prime}$ y longitud $70^{\circ} 0$ ' $25^{\prime}$ ' y en un área 
aproximada de $1088 \mathrm{~m}^{2}$. En esta zona se presenta una época seca de seis meses, predominando un clima seco variable y una época lluviosa de 6 meses en la que predomina un clima lluvioso variable, con una precipitación promedio mensual de $1444.1 \mathrm{~mm}$, una temperatura promedio mensual de $26.2{ }^{\circ} \mathrm{C}$, una humedad relativa promedio mensual de $78.4 \%$ y una evapotranspiración mensual de 99.9 mm (Gómez-Mena et al., 2008).

\section{Características del suelo}

Previo al establecimiento cultivo, se tomaron muestras aleatorias de suelo y se enviaron al Laboratorio Agroempresarial Dominicana (LAD/JAD) de Higuey para fines de análisis. Las características físico-químicas del suelo fueron las siguientes: $\mathrm{pH}$ de 4.91, conductividad eléctrica

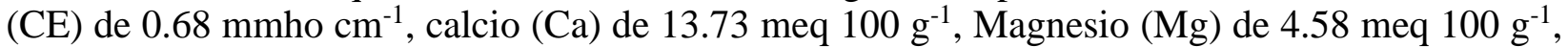
potasio (K) de 0.06 meq $100 \mathrm{~g}^{-1}$, fosforo $(\mathrm{P})$ de $13 \%$, y materia orgánica (MO) de $1.15 \%$.

\section{Establecimiento del cultivo y diseño experimental}

Se utilizaron dos cultivares de semillas de Brachiaria brizantha (Sinaí y Xaraés) a las cuales, previo al experimento se les determinó el porcentaje (\%) de germinación a los 8 y 15 días, donde ambos cultivares presentaron un porcentaje de germinación de 91\% 'Sinaí' y 90\% 'Xaraés', respectivamente. Por otro lado, se procedió a la esterilización del suelo colocándolo a calor directo en un horno (FAITHFUL 101-1AB, China) a una temperatura de $80{ }^{\circ} \mathrm{C}$ durante 30 min, para eliminar microorganismos no deseables en el estudio. Posteriormente, para el establecimiento del experimento se llenaron 24 macetas (capacidad de $3.18 \mathrm{~kg}$ ) con suelo esterilizado y otras 24 con suelo sin esterilizar.

Para todos los tratamientos, se realizó la siembra de manera directa y bien distribuida, colocando 10 semillas por maceta. Para el tratamiento con el hongo micorrízico arbuscular (HMA) Glomus fasciculatum, éste fue sometido previamente a un proceso de conteo directo realizado en el laboratorio de microbiología de la Facultad de Ciencias Agronómicas y Veterinarias de la Universidad Autónoma de Santo Domingo (UASD). El inoculante contenía 11 esporas por cada $100 \mathrm{~g}$ de sustrato, tal como lo reportaron otros estudios (Flores-Juárez et al., 2020). Antes de la siembra, las semillas fueron inoculadas aplicando una dosis de $36 \mathrm{~g}$ del hongo durante un día, para una dosis total de $15 \mathrm{~kg} \mathrm{ha}^{-1}$.

La inoculación fue realizada por recubrimiento de las semillas. Se utilizó un fertilizante mineral (FERQUIDO 20-5-10, República Dominicana) y se aplicó una dosis de $80 \mathrm{~g}^{\text {maceta }^{-1}}$ (150 kg $\mathrm{ha}^{-1}$ ) equivalentes a 50-100-25 de $\mathrm{N}, \mathrm{P}_{2} \mathrm{O}_{5}$ y $\mathrm{K}_{2} \mathrm{O}$ respectivamente. El estiércol fue obtenido de los efluentes del establo bovino y se utilizó una dosis de $100 \mathrm{~g}^{\text {maceta }^{-1}}$ (5 $000 \mathrm{~kg} \mathrm{ha}^{-1}$ ) equivalentes a 33-7-70 de $\mathrm{N}, \mathrm{P}_{2} \mathrm{O}_{5}$ y $\mathrm{K}_{2} \mathrm{O}$. Fue necesaria una reinoculación de las micorrizas con una dosis de $15 \mathrm{~kg} \mathrm{ha}^{-1}$ mezclado con 150 litros de agua (1:10) aplicado vía aspersión al área total, después de cada corte y en época de lluvia.

El experimento se estableció mediante un diseño completamente al azar (DCA) con arreglo factorial $2 \times 2 \times 3$, con cuatro repeticiones: primer factor cultivares [Sinaí y Xaraés], segundo factor suelo [con esterilización $(\mathrm{C})$ y sin esterilización $(\mathrm{S})$ ] y tercer factor tipos de fertilización [micorriza (M), estiércol de bovino (E) y fertilizante químico (Q)]. La descripción del experimento es la: área de planta $=500 \mathrm{~cm}^{2}$, unidad experimental $=0.05 \mathrm{~m}^{2}=0.25 \mathrm{~m}$ x $0.2 \mathrm{~m}$, marco de plantación $=20$ plantas $\mathrm{m}^{-2} \mathrm{y}$ área total del experimento $=2.4 \mathrm{~m}^{2}=0.05 \mathrm{~m}^{2} \mathrm{x} 48$. 


\section{Análisis de datos físico morfológicos y bromatológicos}

Para este experimento se realizó un corte de uniformidad a los 45 días del establecimiento de los cultivares. Luego, se realizaron tres cortes: a) el primero fue el 25 de mayo, a los 21 días después de la aplicación de los tratamientos; b) el segundo se realizó a los 42 días (15 de junio de 2016); y c) el tercero a los 63 días (06 de julio de 2016). La cosecha se realizó con tijeras de corte a una altura promedio de 5 centímetros desde el suelo, pesando cada muestra y guardándola en bolsas de plástico para después analizarlas en el laboratorio. Se tomaron datos de altura de plantas con el apoyo de un flexómetro (Stanley ${ }^{\circledR}$ FatMax ${ }^{\circledR}$ H-1842, USA) (cada 21 días después del corte), tasa absoluta de emergencia de hojas, número de hojas, peso fresco y peso seco. Posteriormente, se analizó la calidad nutritiva de los dos cultivares mediante los procedimientos que se explican a continuación.

Para el contenido de proteína cruda (PC), se utilizó el método Kjeldahl (Horwitz, 2000) calculándose el contenido de nitrógeno por la siguiente fórmula: $\% \mathrm{~N}=\frac{\text { Volumen gastado de } \mathrm{H}_{2} \mathrm{SO}_{4} \times 0.1408}{\text { Peso de la muestra }}$. Luego, a partir del contenido de nitrógeno determinado por el método Kjeldahl multiplicando por el factor 6.25, según la siguiente ecuación: $(\%) \mathrm{Pc}=\mathrm{N}(\%)$ x 6.25.

El \% de Ca se determinó a partir de las cenizas, donde se precipita en forma de oxalato de calcio por neutralización con amoníaco. La fórmula utilizada es la siguiente (Cuello et al., 2017):

$\% \mathrm{Ca}=\frac{\mathrm{V} \times \mathrm{N} \text { Meq Ca } \times 100}{\text { Gramos de muestra } \times \text { alícuota }}$. Con relación al porcentaje de fósforo (\%), éste se determinó por calorimetría (MacNaughtan y Farhat, 2008). El contenido de fibra cruda (\%FC) fue determinado a partir de la materia seca, utilizando el método de digestión, secado e incineración (Cuello et al., 2017).

\section{Análisis estadístico}

Los datos fueron analizados mediante el paquete estadístico Minitab (versión 17). Se realizó un análisis de varianza (Anova) para la composición del rendimiento y la composición bromatológica. Posteriormente, se aplicó la prueba de Tukey $(p \leq 0.05)$ para comparación de medias. Se utilizó el modelo estadístico descrito a continuación: $\mathrm{Y}_{\mathrm{ijk}}=\mu+\mathrm{A}_{\mathrm{i}}+\mathrm{B}_{\mathrm{j}}+\mathrm{C}_{\mathrm{k}}+(\mathrm{AB})_{\mathrm{ij}}+(\mathrm{AC})_{\mathrm{ik}}+(\mathrm{BC})_{\mathrm{jk}}+$ $(A B C)_{i j k}+\varepsilon_{i j k}$ Donde: $i=1,2$ (factor $A=$ cultivares); $j=1,2$ (factor $B=$ suelos); $k=1,2,3$ (factor $\mathrm{C}=$ fertilización).

\section{Resultados y discusión}

\section{Altura de planta}

El factor fertilización generó resultados estadísticamente significativos sobre la altura de planta en el primer corte, en donde, las plantas que recibieron estiércol de bovino consiguieron la mayor altura (valor promedio de $96.06 \mathrm{~cm}$ ) respecto a las que tuvieron micorrizas (valor promedio de 84 $\mathrm{cm}$ ) fertilización mineral (valor promedio de $82.43 \mathrm{~cm}$ ) (Figura 1a). Sin embargo, no hubo diferencias significativas para los factores suelo, cultivar, cultivar*suelo; cultivar*fertilización; suelo*fertilización, cultivar*suelo*fertilización. 

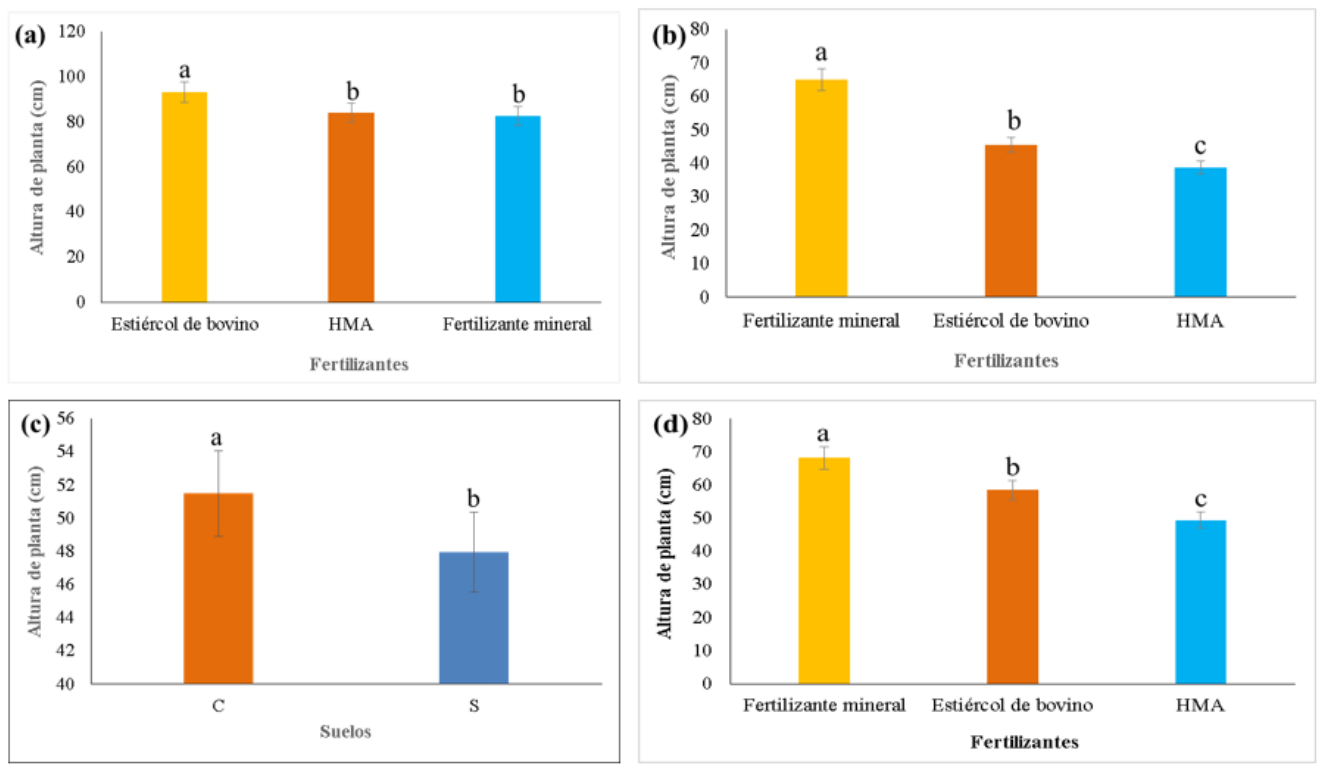

Figura 1. Efecto de la fertilización sobre la altura de dos cultivares de Brachiaria brizantha en a) y b) primer corte; c) segundo corte; y d) tercer corte. Medias con la misma letra para cada figura son estadísticamente iguales (Tukey, $p \leq 0.05$ ).

Los resultados del segundo corte mostraron que hubo diferencias significativas para el tipo de fertilización, siendo el fertilizante mineral con el que se obtuvo la altura promedio más alta de planta $(64.93 \mathrm{~cm})$ y la menor altura de planta la presentó el inoculante HMA $(38.75 \mathrm{~cm})$ (Figura 1b). El factor suelo también generó resultados estadísticamente significativos sobre la altura de planta (Figura 1c). Por otra parte, no hubo diferencias estadísticas significativas para la interacción triple (cultivar*suelo*fertilización), para las interacciones dobles (cultivar*suelo; cultivar*fertilización; suelo*fertilización) y el factor y cultivar.

Para el tercer corte, con la aplicación del fertilizante mineral se obtuvo la mayor altura de las plantas (un promedio de $68.18 \mathrm{~cm}$ ), en comparación al inoculante HMA y al estiércol de bovino (Figura 1d). Por otro lado, no hubo diferencias estadísticas significativas para la interacción triple (cultivar*suelo*fertilizante), ni para las interacciones dobles (cultivar*suelo; suelo*fertilizante; cultivar*fertilizante), tampoco para el factor cultivar, en esta fase del experimento. Los resultados encontrados en este estudio, para la variable altura de planta, fueron mayores a los reportados por González Muñoz et al. (2020), quienes aplicaron un fertilizante mineral en los pastos Insurgente e híbridos de Urochloa, encontraron la altura promedio más alta en plantas del cultivar Cobra (30.9 $\mathrm{cm})$, seguido por el cultivar Mulato II $(28 \mathrm{~cm})$.

La altura menor de plantas, la presentó el cultivar Insurgente $(24 \mathrm{~cm})$. Por otra parte, Flores-Juárez et al. (2020), al inocular el cultivo de avena forrajero con el HMA, encontraron una altura de planta de $25.9 \mathrm{~cm}$. Los HMA tomaron un tiempo para infectar a las raíces de las plantas (Uc-Ku et al., 2019). La aplicación del estiércol de bovino incrementó de manera significativa el pH, y el contenido de materia orgánica (MO); así como, también el fósforo (P) asimilable y potasio (K) intercambiable del suelo (González et at., 2011). Así que, el rendimiento de la variable altura de planta fue influenciado por el estiércol en este estudio. 
Estos resultados confirman el potencial que tiene el estiércol como mejorador de las propiedades químicas y también como fuente de nutrientes para las plantas (Obour et al., 2009; De Souza et al., 2010). En estudios anteriores, se observó que B. brizantha alcanzó mayor altura comparándola con otras especies del mismo género (Pérez et al., 1997). Esas diferencias observadas entre los dos cultivares (Sinaí y Xaraés) en estudio, se deben al hábito de crecimiento de cada uno.

\section{Número de hojas}

Los resultados del primer corte mostraron que no hubo diferencias estadísticas significativas para ninguno de los factores, ni para las interacciones triple y doble con respecto al número de hojas. Esto significa que ni el factor suelo, ni el factor fertilizante influyó en el rendimiento de hojas de los dos cultivares durante este corte. En el segundo corte, el factor fertilización generó resultados estadísticamente significativos sobre el número de hojas, siendo el fertilizante mineral el que generó el más alto número de hojas (promedio fue de 26.69), aplicando (Figura 2a), en comparación a las hojas generadas estiércol y el HMA, los cuales fueron de 20.06 y 18.93, respectivamente.

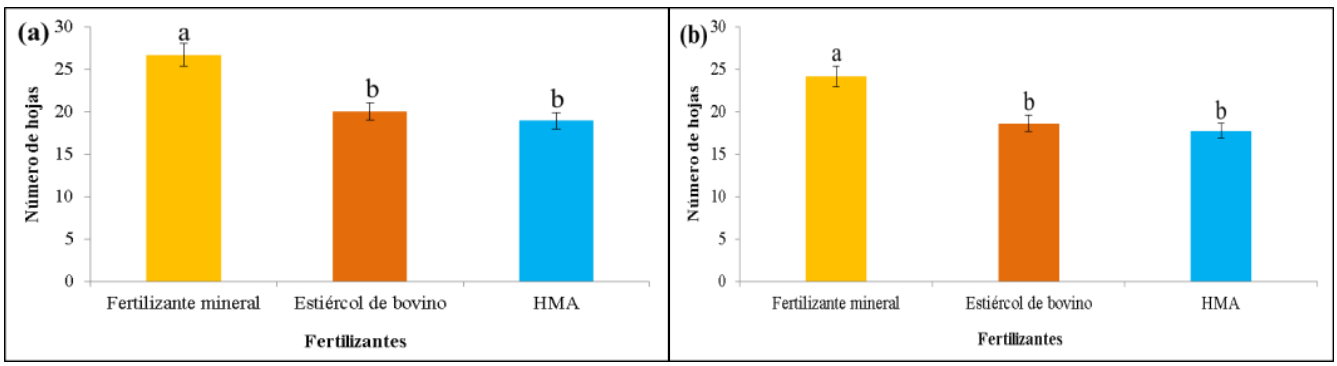

Figura 2. Efecto de la fertilización sobre el número de hojas de dos cultivares de Brachiaria brizantha en a) segundo corte; $\mathbf{y}$ b) tercer corte. Medias con la misma letra para cada figura son estadísticamente iguales (Tukey, $p \leq 0.05$ ).

Para el segundo corte, tampoco hubo diferencias significativas entre las interacciones; ni los efectos principales (suelo y cultivar) mostraron diferencias significativas. Los resultados del tercer corte mostraron que el fertilizante mineral tuvo efecto significativo sobre el número de hojas (Figura 2b), alcanzando un valor promedio de 24.13 en el tercer corte. Esto significó que el fertilizante mineral fue la mejor opción para alcanzar la mayor tasa de emergencia de hojas en los dos cultivares de Brachiaria brizantha. Mientras que, para los factores cultivar, suelo; interacción cultivar*suelo, cultivar*fertilización, suelo*fertilizante, cultivar*suelo*fertilización no hubo diferencias estadísticamente significativas.

Luna et al. (2015), quienes evaluaron la respuesta agronómica de tres variedades de Brachiaria brizantha, encontraron que el cultivar B. mulato logró la mayor producción de follaje respecto a las demás variedades. Este cultivar alcanzó un valor de 774.85 para el número de hojas a los 68 días. Este alto rendimiento se debe al establecimiento del experimento en campo abierto (en 52 parcelas), en comparación a este estudio que se llevó a cabo en macetas. Para Ramírez et al. (2009), la edad de rebrote influye mucho tanto en el crecimiento como también en la calidad de los pastos. 


\section{Rendimiento MV}

Los resultados del primer corte mostraron que no hubo diferencias estadísticas significativas para las interacciones triple y doble sobre el rendimiento de MV. El factor fertilización generó resultados estadísticamente significativos sobre los factores suelo y cultivares. Los resultados también indicaron que el mejor rendimiento de MV se obtuvo con el fertilizante mineral durante los tres cortes; alcanzando valores de $75.62 \mathrm{~g} \mathrm{~m}^{-2}\left(756.2 \mathrm{~kg} \mathrm{ha}^{-1}\right)$ para el primer corte (Figura 3a), $50.1 \mathrm{~g} \mathrm{~m}^{-2}\left(501 \mathrm{~kg} \mathrm{ha}^{-1}\right)$ para el segundo corte (Figura $\left.3 \mathrm{~b}\right)$ y $25.44 \mathrm{~g} \mathrm{~m}^{-2}\left(254.4 \mathrm{~kg} \mathrm{ha}^{-1}\right.$ ) para el tercer corte (Figura 3c), respectivamente en comparación a los tratamientos con estiércol de bovino y el inoculante HMA (Glomus fasciculatum) que resultaron ser iguales en este estudio.

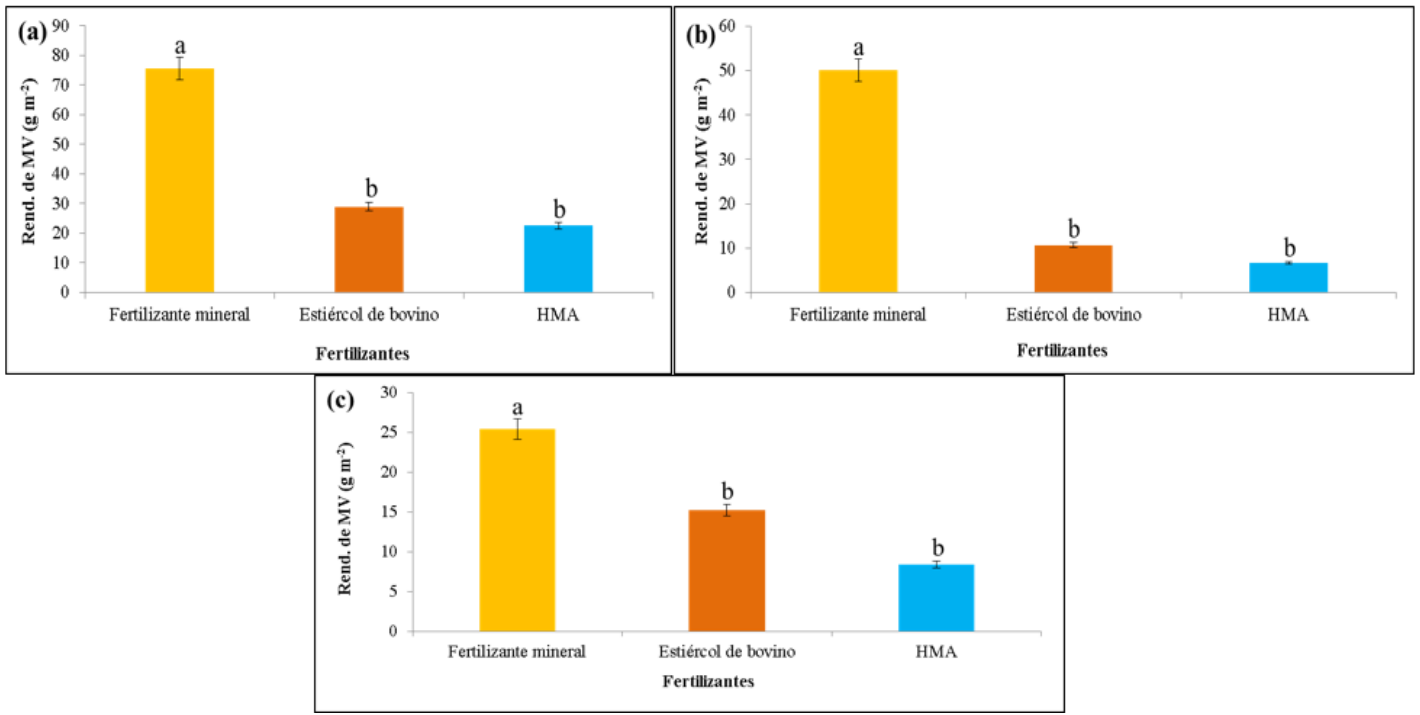

Figura 3. Efecto de la fertilización sobre el rendimiento de MV de dos cultivares de Brachiaria brizantha en a) primer corte; b) segundo corte; y c) tercer corte. Medias con la misma letra para cada figura son estadísticamente iguales (Tukey, $p \leq 0.05$ ). Rend= rendimiento.

Fagundes et al. (2006), quienes evaluaron las características estructurales del pasto Brachiaria en pastizales fertilizado con nitrógeno en las cuatro estaciones, reportaron un rendimiento de MV más alto con respecto a lo encontrado en este estudio. Según estos autores, la aplicación de nutrientes en proporciones adecuadas, puede ser una práctica crucial cuando se pretende incrementar la producción de forraje. La producción de MV disminuyó considerablemente en el tercer corte, esto se debe a la edad del rebrote (Vega et al., 2006; Ramírez et al., 2009).

\section{Rendimiento MS}

En el primer corte, el factor triple cultivar*suelo*fertilizante generó resultados estadísticamente significativos sobre la MS. La mejor combinación (Figura 4a) para obtener el más alto rendimiento de MS (13.5 $\left.\mathrm{g} \mathrm{m}^{-2}\left(132.5 \mathrm{~kg} \mathrm{ha}^{-1}\right)\right)$ lo presentó 'Sinaí' con el fertilizante mineral y el suelo sin esterilizar (Sinaí-Q-S). Mientras el rendimiento más bajo (4.95 $\left.\mathrm{g} \mathrm{m}^{-2}\left(49.5 \mathrm{~kg} \mathrm{ha}^{-1}\right)\right)$, se obtuvo con la combinación del cultivar 'Sinaí' con el inoculante HMA y el suelo sin esterilizar (Sinaí-M-S). La fertilización con el estiércol + el suelo esterilizado, mostró un efecto significativo sobre el rendimiento de MS (11.2 $\left.\mathrm{g} \mathrm{m}^{-2}\left(112 \mathrm{~kg} \mathrm{ha}^{-1}\right)\right)$ del cultivar Sinaí. 


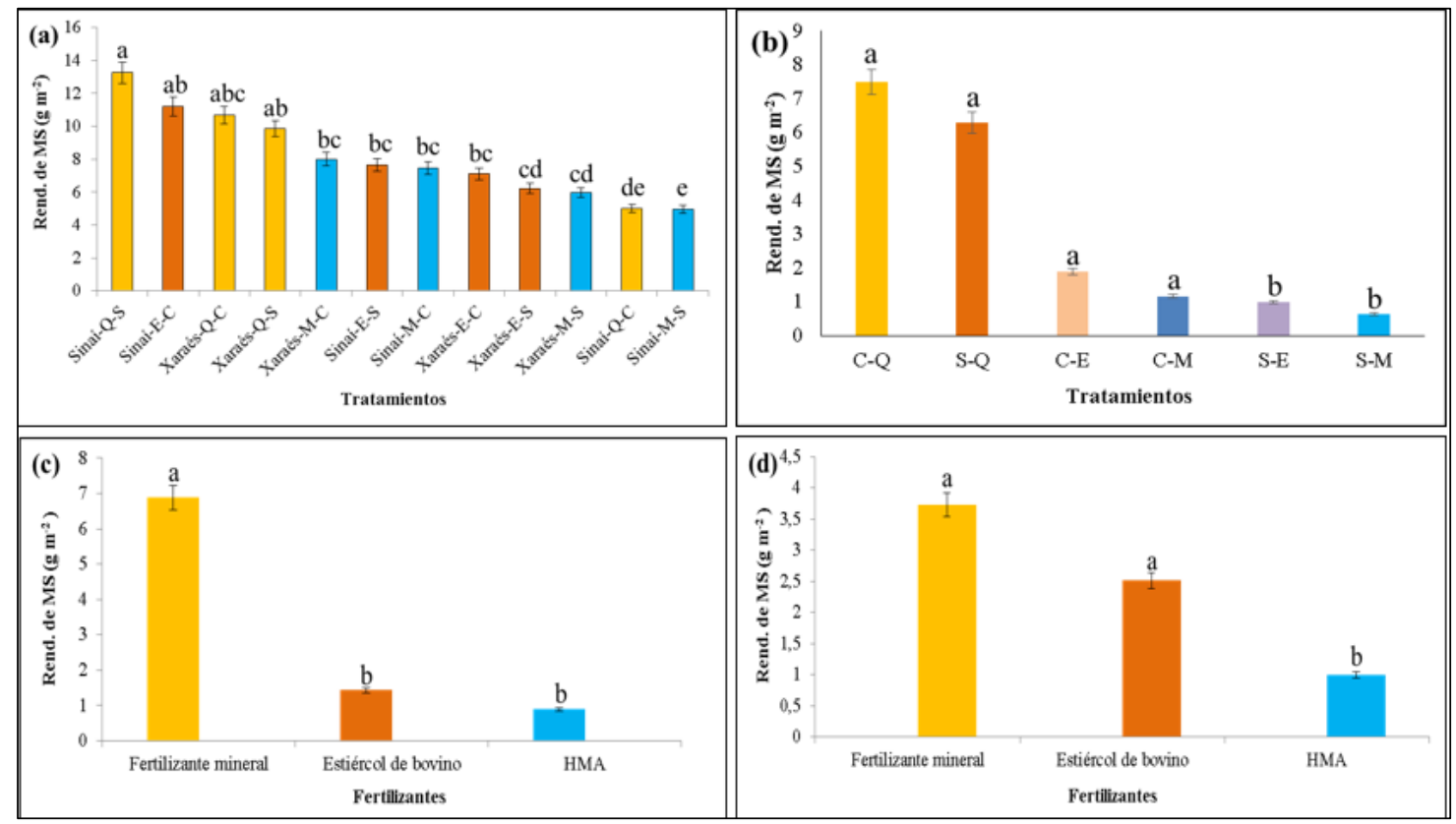

Figura 4. Efecto de la interacción cultivar*suelo*fertilizante en a) y b) primer corte y efecto de la fertilización en c) segundo corte; y d) tercer corte sobre el rendimiento de MS de dos cultivares de Brachiaria brizantha. Medias con la misma letra para cada figura son estadísticamente iguales (Tukey, $p \leq 0.05$ ). $\mathrm{Q}=$ fertilizante mineral; $\mathrm{S}=$ suelo $\sin$ esterilizar; $\mathrm{C}=$ suelo esterilizado; $\mathrm{M}=$ hongo micorrízico arbuscular; Rend= rendimiento.

Debido a la combinación del fertilizante mineral + el suelo esterilizado, 'Xaraés' alcanzó un rendimiento de $10.67 \mathrm{~g} \mathrm{~m}^{-2}\left(106.7 \mathrm{~kg} \mathrm{ha}^{-1}\right)$ de MS. Para el factor doble suelo*fertilizante hubo diferencias estadísticamente significativas entre los tratamientos. Los dos tratamientos que tuvieron mejores rendimientos (Figura $4 \mathrm{~b}$ ) fueron $\mathrm{C}-\mathrm{Q}$ (promedio de $7.5 \mathrm{~g} \mathrm{~m}^{-2}$ ) y S-Q (promedio de $6.28 \mathrm{~g} \mathrm{~m}^{-2}$ ). Se puede apreciar que el fertilizante mineral tuvo efecto muy significativo sobre el rendimiento de MS para los cultivares, en comparación a las demás fuentes de fertilización. En el segundo corte el factor fertilizante produjo resultados estadísticamente significativos sobre el contenido de MS. En la Figura 4c se observa que la aplicación del fertilizante mineral logró el mejor rendimiento de MS con un valor de $6.88 \mathrm{~g} \mathrm{~m}^{-2}\left(68.8 \mathrm{~kg} \mathrm{ha}^{-1}\right)$, seguido por el estiércol (1.43 $\left.\mathrm{g} \mathrm{m}^{-2} ; 14.3 \mathrm{~kg} \mathrm{ha}^{-1}\right)$ y el inoculante HMA $\left(0.89 \mathrm{~g} \mathrm{~m}^{-2} ; 8.9 \mathrm{~kg} \mathrm{ha}^{1}\right)$, respectivamente. Por el contrario, las interacciones triple y doble, el factor principal cultivar y suelo generaron resultados estadísticamente no significativos sobre el contenido de MS.

En el tercer corte el factor fertilizante produjo resultados estadísticamente significativos sobre el contenido de MS. La aplicación del fertilizante mineral mostró el mejor rendimiento de MS, logrando una producción de $3.73 \mathrm{~g} \mathrm{~m}^{-2}\left(37.3 \mathrm{~kg} \mathrm{ha}^{-1}\right)$ (Figura 4d). Esta disminución del rendimiento de MS registrada en este estudio (durante los cortes 1 y 2), se debió a la falta de lluvia durante el rebrote y también a la edad de los pastos.

Los resultados obtenidos en los dos cultivares de pastos analizados con relación al rendimiento de MS, fueron más bajos con respecto a los reportados por Luna et al. (2015), quienes encontraron valores de $480 \mathrm{~kg} \mathrm{ha}^{-1}\left(0.48 \mathrm{t} \mathrm{ha}^{-1}\right)$ a los 21 días (primer corte), $690 \mathrm{~kg} \mathrm{ha}^{-1}\left(0.69 \mathrm{t} \mathrm{ha}^{-1}\right)$ a los 42 
días (segundo corte) y $1240 \mathrm{~kg} \mathrm{ha}^{-1}\left(1.24 \mathrm{t} \mathrm{ha}^{-1}\right)$ a los 63 días (tercer corte), respectivamente. Estos autores ante mencionados reportaron valores similares a los encontrados por Fernández et al. (2004); Reyes et al. (2009), quienes evaluaron diferentes cultivares de Brachiaria y Panicum en diferentes condiciones ambientales. Por otro lado, Garay et al. (2017) reportaron rendimiento más alto $\left(5.06 \mathrm{t} \mathrm{ha}^{-1}\right)$ en el $c v$ Xaraés; en comparación a lo reportado en este estudio.

\section{Composición bromatológica}

\section{Contenido de PC}

En el Cuadro 1 se observan los contenidos promedio de PC, Ca, P, FC de dos cultivares de Brachiaria brizantha, donde el mayor contenido de PC se encontró en las plantas de ambas variedades que recibieron fertilización química sin importar si tuvieron un suelo esterilizado o no y las menores concentraciones existieron en ambos cultivares que recibieron micorrizas sin importar si el suelo presentó esterilización o no desde un inicio. Los resultados de los factores cultivar, suelo y fertilización, así como también las interacciones dobles cultivar*suelo, cultivar*fertilizante, suelo*fertilizante fueron estadísticamente no significativos.

Cuadro 1. Composición química promedio de dos cultivares de Brachiaria brizantha estudiados en relación con el efecto de la interacción cultivar*suelo*fertilización.

\begin{tabular}{ccccc}
\hline Tratamientos & PC & Ca & P & FC \\
\hline Sinaí-E-C & $10.67 \mathrm{~b}$ & $1.62 \mathrm{c}$ & $0.26 \mathrm{c}$ & $27.27 \mathrm{a}$ \\
Sinaí-Q-C & $20.81 \mathrm{a}$ & $1.69 \mathrm{bc}$ & $0.27 \mathrm{~b}$ & $20.59 \mathrm{c}$ \\
Sinaí-M-C & $8.63 \mathrm{c}$ & $1.89 \mathrm{a}$ & $0.34 \mathrm{a}$ & $25.15 \mathrm{bc}$ \\
Sinaí-E-S & $9.87 \mathrm{~b}$ & $1.9 \mathrm{a}$ & $0.35 \mathrm{~b}$ & $21 \mathrm{c}$ \\
Sinaí-Q-S & $18.19 \mathrm{a}$ & $1.67 \mathrm{~b}$ & $0.3 \mathrm{c}$ & $21.8 \mathrm{~b}$ \\
Sinaí-M-S & $10.75 \mathrm{~b}$ & $1.63 \mathrm{~b}$ & $0.37 \mathrm{a}$ & $24.61 \mathrm{a}$ \\
Xaraés-E-C & $11.29 \mathrm{bc}$ & $1.72 \mathrm{a}$ & $0.3 \mathrm{~b}$ & $23.64 \mathrm{bc}$ \\
Xaraés-Q-C & $19.85 \mathrm{a}$ & $1.37 \mathrm{~b}$ & $0.34 \mathrm{~b}$ & $22.39 \mathrm{c}$ \\
Xaraés-M-C & $10.31 \mathrm{~b}$ & $1.62 \mathrm{a}$ & $0.37 \mathrm{a}$ & $27.1 \mathrm{a}$ \\
Xaraés-E-S & $10.08 \mathrm{~b}$ & $1.72 \mathrm{~b}$ & $0.28 \mathrm{c}$ & $23.74 \mathrm{bc}$ \\
Xaraés-Q-S & $18.81 \mathrm{a}$ & $1.47 \mathrm{~b}$ & $0.35 \mathrm{~b}$ & $21.69 \mathrm{c}$ \\
Xaraés-M-S & $9.79 \mathrm{c}$ & $2.08 \mathrm{a}$ & $0.42 \mathrm{a}$ & $25.33 \mathrm{a}$ \\
Rango adecuado & $4-18$ & 0.3 & 0.3 & $<50$ \\
\hline
\end{tabular}

Medias con la misma letra en la misma columna son estadísticamente iguales (Tukey, $p \leq 0.05$ ). $\mathrm{PC}=$ proteína cruda; $\mathrm{Ca}=$ calcio; $\mathrm{P}=$ fósforo; $\mathrm{FC}=$ fibra cruda; $\mathrm{E}=$ estiércol de bovino; $\mathrm{M}=$ micorriza; $\mathrm{Q}=$ fertilizante mineral; $\mathrm{S}=$ suelo sin esterilizar; $\mathrm{C}=$ suelo esterilizado.

El contenido de PC obtenido en ambos cultivares fue superior a lo reportado encontrado en otros estudios, utilizando Brachiaria brizantha cv Mulato II. Se encontraron valores de PC de $14.5 \%$ (Garay et al., 2017; Gonzales Muñoz et al., 2020) y 15\% (Castillo et al., 2006), respectivamente. En este estudio, los valores más altos de PC fueron ligeramente superiores a los rangos adecuados (Vargas González, 1989). Sin embargo, los valores menores encontrados fueron dentro o iguales a 
los rangos adecuados (Cuadro 1). Jiménez et al. (2010), quienes reportaron que cualquier tipo de fertilización influye sobre la PC. Lo que se confirmó en este estudio. Bernal (1994) reportaron que al aumentar la madurez de los forrajes el valor proteico disminuye. Luego, Garay et al. (2017) reportaron que el factor tiempo influye en la disminución del contenido de PC.

\section{Contenido de Ca y $\mathbf{P}$}

El mayor contenido de Ca (Cuadro 1) se encontró en las plantas de Sinaí y Xaraés que recibieron fertilización orgánica sin esterilizar y las menores concentraciones existieron en plantas Xaraés que recibieron fertilizante inorgánico sin importar si el suelo presentó esterilización o no desde un inicio. El mayor contenido de $\mathrm{P}$ se encontró en la planta Xaraés que recibió micorriza en suelo sin esterilizar, y la menor concentración existió en la planta Sinaí que recibió estiércol con suelo esterilizado desde un inicio.

En general, los forrajes son una buena fuente de Ca. Según Minson (1990), el contenido de Ca que presentan los forrajes se ven influenciados por la especie, porción consumida de planta; así como también, madurez, cantidad de calcio intercámbiale en el suelo y el clima. El contenido de Ca reportado en ambos cultivares fue superior a los rangos adecuados (Vargas-González, 1989).

\section{Contenido de FC}

El mayor contenido de FC (Cuadro 1) se encontró en la planta Sinaí que recibió estiércol de bovino en suelo esterilizado, así como también en la planta Xaraés que recibió micorriza en suelo esterilizado y la menor concentración existió en la planta Sinaí que recibió fertilizante químico con suelo esterilizado desde un inicio. Los resultados obtenidos en este estudio sugieren que independientemente de las diferencias estadísticas en la composición bromatológica, los cultivares mostraron características nutricionales adecuadas y constituyen una alternativa para los sistemas silvopastoriles (Bugarín et al., 2009).

\section{Conclusiones}

El contenido de materia seca fue influenciado por los tres factores estudiados, donde éstos resultaron ser diferentes. De esa manera, la producción promedio de materia seca más alta (132.5 $\mathrm{kg} \mathrm{ha}^{-1}$ ) fue lograda en el $c v$ Sinaí a los 21 días. La mejor combinación para la obtención este rendimiento fue el fertilizante mineral con el suelo sin esterilizar. Por otro lado, el tipo de fertilización influyó sobre la calidad nutricional y el rendimiento de follaje de los dos cultivares. El tiempo de corte fue uno de los factores más importantes, se observó que, a menor tiempo de corte, la cantidad de nutrientes en los pastos aumenta de manera frecuente, ya que a los 21 días después del rebrote se encontraron mayor concentración de elementos nutritivos.

Por otro lado, el mayor contenido promedio de PC se encontró en las plantas de ambas variedades que recibieron fertilización química. El mayor contenido promedio de Ca se encontró en Sinaí y Xaraés que recibieron fertilización orgánica. Mientras que el mayor contenido promedio de $\mathrm{P}$ fue encontrado en Xaraés con la aplicación de HMA. Finalmente, la planta Sinaí que recibió estiércol de bovino en suelo esterilizado presentó mayor contenido promedio de FC. Este estudio afirma que la fertilización cualquiera que sea tiene un efecto significativo en la producción de forraje verde y también materia seca. 


\section{Agradecimientos}

El autor principal agradece al Consejo Nacional de Ciencia y Tecnología (CONACYT) por la beca de los estudios de doctorado; así como, la OEA a través del programa de becas CONACYT-OEAAMEXCID por el apoyo único otorgado. Wilgince Apollon e Yviane Jean-Baptiste, agradecen de manera particular a la Mtra. Birmania Wagner por su asesoría en la realización del proyecto. Finalmente, Wilgince Apollon agradece al PhD Alejandro Isabel Luna-Maldonado y Dr. Héctor Silos-Espino por su apoyo incondicional.

\section{Literatura citada}

Bernal, E. 1994. Pastos y forrajes tropicales. Producción y manejo. Bogotá, DC. (Col). Departamento de publicaciones del banco ganadero. $3^{\mathrm{a}}$ (Ed.). Bogotá, DC. Colombia. $575 \mathrm{p}$.

Bogdan, A. V. 1977. Tropical pasture and fodder plants. Longman. 475 p.

Bugarín, J.; Lemus, C.; Sangines, L.; Aguirre, J.; Ramos, A.; Soca, M. y Arece, J. 2009. Evaluación de dos especies de leucaena, asociadas a Brachiaria brizantha y Clitoria ternatea en un sistema silvopastoril de Nayarit, México. Producción y composición bromatológica de la biomasa. Pastos y Forrajes. 32(4):1-9. http://scielo.sld.cu/pdf/pyf/v32n4/pyf07409.pdf.

Castillo, M.; Vélez, M.; Rosas, J. C. y Trabanino, R. 2006. Producción y composición de los cultivares Mulato I y II de Brachiaria híbrido inoculados con micorriza y Trichoderma harzianum. Ceiba. 47(1-2):25-32. https://doi.org/10.5377/ceiba.v47i1-2.444.

Cuello, M.; Jaramillo, G. K.; Canchingre, E.; Pérez, J. C.; Castro, C. y Cabrera, O. 2017. Determinación de componentes nutricionales presentes en las hojas secas de Annona muricata L. (Guanábana). Rev. Cumbre. 3(1):9-16. https://dialnet.unirioja.es/servlet/ articulo?codigo $=6550744$.

Da Silva-Souza, F. A. P.; Dutra, S. y Serrão, E. A. S. 1992. Productivadade estacional e composição quimica de Brachiaria humidicola e pastagem nativa de campo cerrado do estao de Amapá, Brasil. Pasturas Tropicales. 14(1):11-16. https://ainfo.cnptia.embrapa.br/digital/bitstream /item/43138/1/Vol14-rev1-ano92-art3.pdf.

De Souza, R. F.; Faquin, V.; Sobrinho, R. R. L. y Oliveira, E. A. B. 2010. Influência de esterco bovino e calcário sobre o efeito residual da adubação fosfatada para a Brachiaria brizantha cultivada após o feijoeiro. Rev. Bras. Cienc. Sol. 34(1):143-150. https://doi.org/10.1590 /S0100-06832010000100015.

Fagundes, J. L.; Fonseca, D. M.; Mistura, C.; Morais, R. V.; Teixeira-Vitor, C. M.; Gomide, J. A.; Nascimento-Junior, D.; Casagrande, D. C. y Costa, L. T. 2006. Características morfogênicas e estruturais do capim-braquiária em pastagem adubada com nitrogênio avaliadas nas quatro estações do ano. Rev. Brasil. Zoot. 35(1):21-29. https://doi.org/10. 1590/S1516-35982006000100003.

Fernández, J. L.; Benítez, D. E.; Gómez, I.; de Souza, A. y Espinosa, R. 2004. Rendimiento de materia seca y contenido de proteína bruta del pasto Panicum maximum vc likoni en un suelo Vertisol de la provincia Granma. Rev. Cubana. Cienc. Agríc. 38(4):417-421. https://www.redalyc.org/articulo.oa?id=193017793013.

Flores-Juárez, D.; Villegas-Aparicio, Y.; Castro-Rivera, R.; Gómez-Vázquez, A.; CarrilloRodríguez, J. C. y Castañeda-Hidalgo, E. 2020. Inoculación de avena forrajera con hongos micorrízicos arbusculares. Rev. Mex. Cienc. Agríc. 24(esp.):191-199. https://doi.org/ 10.29312/remexca.v0i24.2369. 
MacNaughtan, B. and Farhat, I. A. 2008. Thermal methods in the study of foods and food ingredients. In: Gabbott, P. (Ed.). Principles and applications of thermal analysis. WileyBlackwell. 330-409. https://doi.org/10.1002/9780470697702.ch2; https://doi.org/10.1002/ 9780470697702.ch9.

Garay, J. R.; Joaquín, S.; Zárate, P.; Ibarra, M. A.; Martínez, J. C.; González, R. P. and Cienfuegos, E. G. 2017. Acumulación de materia seca y concentración de proteína cruda en cultivares de Brachiaria spp. En el trópico húmedo de Ecuador. Tropical Grassland Forrajes Tropicales. 2(5):66-76. http://dx.doi.org/10.17138/tgft(5)66-76.

Gómez-Mena, J.; Julia-Mera, E.; Meyreles, L. y Portorreal, F. 2008. Historia natural y social del Río Haina; primera parte: características físico-naturales de la cuenca Haina. Ciencia y Sociedad. 33(4):475-494. http://dx.doi.org/10.22206/cys.2008.v33i4.pp475-94.

González-Muñoz, A.; Garay-Martínez, J.; Estrada-Drouaillet, B.; Bernal-Flores, Á.; LimasMartínez, A. y Joaquín-Cancino, S. 2020. Rendimiento y contenido de proteína en forraje y ensilado de pasto Insurgente e híbridos de Urochloa. Rev. Mex. Cienc. Agríc. 24(esp.):177-189. https://doi.org/10.29312/remexca.v0i24.2368.

González, P. J.; Rivera, R.; Arzola, J.; Morgan, O. y Ramírez, J. F. 2011. Efecto de la inoculación de la cepa de hongo micorrízico arbuscular Glomus hoi-like en la respuesta de Brachiaria híbrido $c v$ Mulato II (CIAT 36087) a la fertilización orgánica y nitrogenada. Cultivos Tropicales. 32(4):05-12. http://scielo.sld.cu/pdf/ctr/v32n4/ctr01411.pdf.

Havlin, J. L; Tisdale, S. L; Nelson, W. L. and Beaton, J. D. 2005. Soil fertility and fertilizers: an introduction to nutrient management. Pearson, $7^{\text {th }}$ (Ed.). New Jersey, U. S. A. 515 p.

Horwitz, W. 2000. Official methods of AOAC international. Association of official analytical chemists (AOAC) international, 17 ${ }^{\text {th }}(\mathrm{Ed})$. Gaithersburg. USA. 200 p.

Jiménez, O. M. M.; Granados, L.; Oliva, J.; Quiroz, J. y Barrón, M. 2010. Calidad nutritiva de Brachiaria humidicola con fertilización orgánica e inorgánica en suelos ácidos. Arch. Zootec. 59(228):561-570. http://scielo.isciii.es/pdf/azoo/v59n228/art9.pdf.

Lascano, C.; Pérez, R.; Plazas, C.; Medrano, J.; Pérez, O. y Argel, P. J. 2002. Pasto Toledo. Cali, Colombia. Corporación Colombiana de investigación agropecuaria. Centro internacional de agricultura tropical (CIAT). Cali, Colombia. 22 p. http://ciat-library.ciat.cgiar.org/ articulos_ciat/brachiaria_brizantha_cv_toledo.pdf.

Lok, S.; Crespo, G. y Torres, V. 2017. Influencia de las leguminosas forrajeras en el sistema suelopasto. Cuban J. Agric. Sci. 51(2):261-270. http://scielo.sld.cu/pdf/cjas/v51n2/cjas 12217.pdf.

Luna, R. A.; Pérez, J. J.; Avellaneda, J. H.; Espinoza, A. L.; Iza, N. B. y Luna, M. V. 2015. Respuesta agronómica de tres variedades de Brachiaria en el cantón el empalme provincia del Guayas. Ecuador. Cienc. Tecnol. 8(2):45-50. https://www.uteq.edu.ec/revistacyt/ publico/archivos/C2_V8\%20N2\%205Luna\%20et\%20al.pdf.

Minson, D. 1990. Forage in ruminant nutrition. Academic press, San Diego. 502 p. https://doi.org/ 10.1016/B978-0-12-498310-6.X5001-9.

Moura, L. O.; Braga, C. M.; Bastos-Veiga, J. y Amador-Costoa, N. 2002. Avaliação de pastagem de quicuio-da-amazônia (Brachiaria humidicola (Rendle) Schweickerdt) en sistema de pastejo rotacionado intensivo, en Belén, Pará. Pasturas Tropicales. CIAT. 24(2):30-39. http://ciat-library.ciat.cgiar.org/Articulos_Ciat/Vol_24_02_05.pdf.

Obour, A. K.; Silveira, M. B.; Adjei, J.; Vendramini, M. y Rechcigl, J. E. 2009. Cattle manure application strategies effects on bahiagrass yield, nutritive value, and phosphorus recovery. Agron. J. 101:1099-1107. https://doi.org/10.2134/agronj2009.0006. 
Pérez, S.; Faria, M. y González, B. 1997. Evaluación agronómica de gramíneas forrajeras en Carora, Estado Lara, Venezuela. Rev. Facul. Agron. Universidad del Zulia. 16(6):621-636. https://produccioncientificaluz.org/index.php/agronomia/article/view/26289/26915.

Ramírez, O.; Hernández, A.; Carneiro, S.; Pérez, J.; Enríquez, J. F.; Quero, A.; Guadalupe, J.; Herrera, H. y Cervantes, A. 2009. Acumulación de forraje, crecimiento y características del pasto mombasa (Panicum maximum Jacq.). Rev. Téc. Pec. 47(2):203-213. https://www.redalyc.org/articulo.oa?id=61312116008.

Reyes, P. A.; Bolaños-Aguilar, E. D.; Hernández-Sánchez, D.; Aranda-lbañez, E. M. e IzquierdoReyes, F. 2009. Producción de materia seca y concentración de proteína en 21 genotipos del pasto humidícola Brachiaria humidicola (Rendle) Schweick. Universidad y Ciencia. 25(3):213-224. https://www.redalyc.org/articulo.oa?id=15416281003.

Uc-Ku, A.; Arreola Enríquez, J.; Carrillo Ávila, E.; Osnaya González, M.; Alarcón, A.; Ferrera Cerrato, R. y Landeros Sánchez, C. 2019. Inoculación de hongos micorrízicos arbusculares en el cultivo de Heliconia stricta. Rev. Mex. Cienc. Agríc. 10(5):1057-1069. https://doi.org/10.29312/remexca.v10i5.1608.

Vargas-González, E. 1989. Contenido mineral y proteico para rumiantes en pastoreo en Costa Rica1. Editorial de la Universidad de Costa Rica, 1. (Ed.). San José, C. R. 217 p.

Vega, E.; Ramírez, J. L.; Leonard, I. e Igarza, A. 2006. Rendimiento, caracterización química y digestibilidad del pasto Brachiaria decumbens en las actuales condiciones edafoclimáticas del Valle del Cauto. Rev. Electrónica de Veterinaria. 7:1-6. http://www.veterinaria. org/revistas/redvet. 